\title{
Compressed Sensing Imaging for Staggered SAR with Low Oversam- pling Rate
}

\author{
Xingxing Liao ${ }^{(\bowtie) a}$, Changlin Jin ${ }^{\mathrm{b}}$, and Zhe Liu ${ }^{\mathrm{a}}$ \\ ${ }^{a}$ University of Electronic Science and Technology of China, Chengdu, China, liaonay@ std.uestc.edu.cn \\ ${ }^{\mathrm{b}}$ Southwest China Research Institute of Electronic Equipment, Chengdu, China
}

\begin{abstract}
This paper focuses on processing low oversampling echo data of staggered synthetic aperture radar (SAR). In staggered mode, the non-uniformly sampling and irregular loss of echo data cause azimuth ambiguity which severely degrades the imaging quality. To solve this problem, we propose a compressed sensing (CS) method in which the non-uniform fast Fourier transform (NUFFT) technique is adopted to obtain uniform azimuth spectrum, and the fast iterative shrinkage thresholding algorithm (FISTA) is utilized to efficiently reconstruct the ambiguity-free image from in-complete echo data. Simulation results demonstrate the proposed method can effectively suppress the azimuth ambiguity in the vicinity of targets.
\end{abstract}

Keywords: Staggered SAR, low oversampling, compressed sensing (CS), NUFFT

\section{Introduction}

Synthetic Aperture Radar (SAR) is well proven to be an important earth observation radar, independent of adverse weather conditions and capable of working at both day and night. Since in the duration in which conventional SAR is receiving echo data, it must close the receiving subsystem, blind ranges along range direction will occur. Furthermore, in conventional SAR mode, because the PRI is invariable, blind ranges remain unchanged during the whole synthetic aperture. The width of the blind range is big enough in high-orbit SAR platform so that it cannot be omitted.

In order to get the coverage of a continuous large swath, staggered SAR concept has been come up with by authors in [2]. During the acquisition process of the staggered SAR system, the pulse repetition interval (PRI) changes, resulting in the blockage to move along the swath Figure $\mathbf{1}$ and Figure 2. Nevertheless, staggered SAR imaging is confronted with two unavoidable problems: non-uniform sampling in azimuth and the loss of echo impulses.

Strategies in [1]-[3] have successfully solved these two problems, but they both need to adopt large over-sampling rates. The best linear unbiased (BLU) estimation method is proposed in [2] to well utilize the knowledge of its power spectral density so as to recover the missing information at certain positions. The recovering accuracy of BLU algorithm depends highly on system PRF. In [3], an improved multichannel reconstruction algorithm based on a linear constraint minimum power (LCMP) method has been proposed to improve the imaging quality with high oversampling rate. High oversampling rates are essential to lower ambiguity levels in staggered SAR, but it is undesirable for the use of radar system in space due to data-rate constraints. Therefore, many works, [4]-[6], now

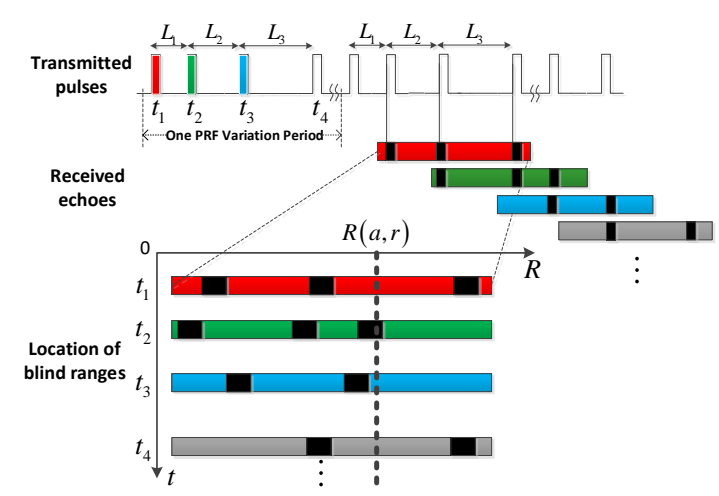

Figure 1 : Location of blind ranges along range distance in staggered SAR mode.

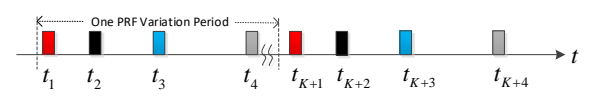

Figure 2 : Received echo data corresponding to target at $(a, r)$ in Figure $\mathbf{1}$ which is non-uniformly spaced and has the lost samples marked in black.

focus on staggered SAR imaging with low oversampling rates to obtain high azimuth resolution. In [4], to address the ambiguity occurs in azimuth in staggered SAR mode with low over-sampling rates, a method combines missing data iterative adaptive algorithm (MIAA) with multichannel reconstruction based on minimizing ambiguity energy is proposed to eliminate the ambiguity in azimuth. This method takes advantage of MIAA as a CS algorithm which can be used to recover the missing echo data of targets even if when they are under sub-Nyquist sampling rate. In [5], a method is proposed to apply to both point-like and distributed scatters sampled in staggered mode under 
low oversampling rate. On the basis of [4], this method adds a step to determine whether the scenic spot is a distributed target. If the result is a distributed target, BLU estimation algorithm is directly performed on the radar echo data; otherwise, it is processed according to the method in [4]. However, both [4] and [5] take the accurate recovery of the sample loss as a prerequisite for acquiring a high resolution focused image. Besides, targets inside different blind ranges have different loss patterns, which means that during the missing echo data recovering process, careful consideration should be taken into the adaptation of certain parameters due to the complicatedly changed blind ranges. In this paper, a CS strategy based on NUFFT and FISTA is proposed to improve the imaging quality of staggered SAR with low oversampling rates. First, using NUFFT in azimuth, the proposed method can obtain the uniform frequency samples in azimuth. Second, the proposed method circumvents the recovery of the missing samples by exploiting the merits of CS strategy, which can well deal with sub-Nyquist SAR data, as shown in [7], [8]. Plus, our method is able to suppress ambiguity caused by missing samples and non-uniform sampling at the same time by adopting FISTA, as used in [8], to solve the constructed optimization problem.

\section{Signal processing method}

\subsection{Construction of signal model}

The staggered SAR system adopting changing PRIs to make the blockage dispersedly move along the swath. A linear PRI trend ranging in the interval $\left[P R I_{\min }, P R I_{\max }\right]$ has the advantage of letting the system designer optimize the choice of the PRIs in relation to other system parameters and control the location of the resulting gaps. The periodic variable PRI can be defined as

$$
L_{k+1}=L_{k}-\Delta, k=1, \ldots, K-1
$$

where $L_{1}=P R I_{\max }, L_{K}=L_{1}-(K-1) \Delta=P R I_{\min }$; $K$ is the number of PRIs in one PRI linear variation period. Therefore, the sampling slow time of staggered SAR can be expressed as

$$
t_{n}=t_{1}+\operatorname{floor}(n / N) \cdot \sum_{i=1}^{K} L_{k}+\sum_{i=1}^{\bmod (n-1, K)} L_{k}
$$

where $t_{1}$ is when staggered SAR system starts transmitting pulse; $n$ is the index of the transmitted pulses, $n=$ $1, \ldots, N_{a}$, and $N_{a}$ is the total number of transmitting pulses; floor $(\epsilon)$ is the function that rounds the element of epsilon to the nearest integer towards minus infinity; mod $\left(\epsilon_{1}, \epsilon_{2}\right)$ is the function which returns $\epsilon_{1}-$ floor $\left(\epsilon_{1}, \epsilon_{2}\right)$. To process the staggered-SAR echo data, we need to know the positions of missing samples caused by staggered mode. Due to that the loss pattern of a target's echo data sampled in staggered mode is decided by range distance of the target, we can easily distinguish the losing patterns of targets in different blind ranges after range compression process. Therefore, we first perform range compression

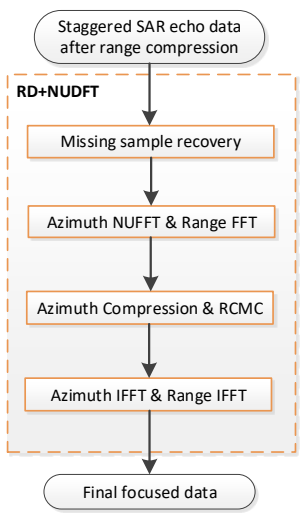

(a)

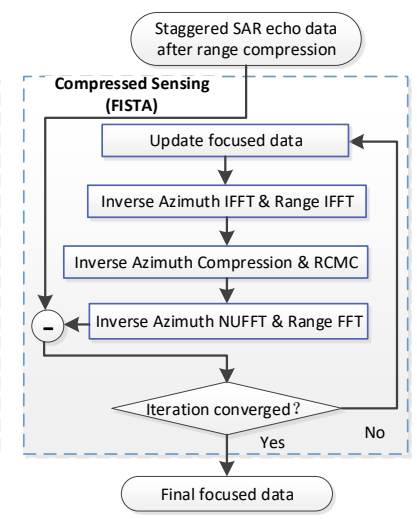

(b)
Figure 3 : The flowcharts of different processing strategies for staggered SAR data. (a) Previous method based on re-covering missing samples. (b) Proposed method based on CS without the process of recovering missing data.

on raw data in the range-frequency and azimuth-time domains. Then the sampled staggered SAR signal for a point target at $(a, r)$ can be described as follows:

$$
\begin{array}{r}
S_{r c}[n, m]=\sigma_{1} B[n, m] w_{r}\left(\tau_{m}-\frac{2 R(n)}{c}\right) w_{a}\left(t_{n}-t_{c}\right) \\
\delta\left(\tau_{m}-\frac{2 R(n)}{c}\right) \exp \left(-\frac{j 4 \pi f_{0} R(n)}{c}\right)
\end{array}
$$

where $B[n, m]$ denotes the missing information of $S_{r c}[n, m] ; w_{r}(\cdot)$ and $w_{a}(\cdot)$ denote the antenna pattern functions in the range and azimuth directions, respectively; $\sigma_{1}$ represents the scattering coefficient of the target at (a,r); $c$ is the speed of light; $t_{n}$ is the $n_{t h}$ sampling slow time in azimuth given in (2), $n=1, \ldots, N_{a} ; \tau_{m}$ is the $m_{t h}$ sampling fast time in range, $m=1, \ldots, N_{r} ; t_{c}$ is the Doppler center time; $f_{0}$ is the carrier frequency; $R(n)$ is the distance of the target at $(a, r)$ from radar at the sampling slow time $t_{n}$.

\subsubsection{Resampling method}

In staggered SAR mode, because of the non-uniform sampling in azimuth, directly adopting FFT to transform the time domain data into frequency domain data in azimuth will cause strong azimuth ambiguity in the final focused image. As seen in Figure 3(a), we replace FFT with NUFFT in azimuth within the range doppler algorithm (RDA) to solve this problem, and without considering the missing samples, the imaging process of staggered SAR raw data can be formulated as

$$
I^{\prime}=F_{a}^{-1}\left(A \odot\left(F_{N_{-} a} S_{r c} F_{r}\right)\right) F_{r}^{-1}
$$

where $A \in \mathbb{C}^{N_{a} \times N_{r}}$ de-notes azimuth compression and approximate range cell migration correction (RCMC); $F_{N_{-} a}, F_{a}^{-1} \in \mathbb{C}^{N_{a} \times N_{a}}$ are respectively the azimuth NUDFT and IDFT matrix; $F_{r}, F_{r}^{-1} \in \mathbb{C}^{N_{r} \times N_{r}}$ are respectively the range DFT and IDFT matrix; $\odot$ is the Hardmard 


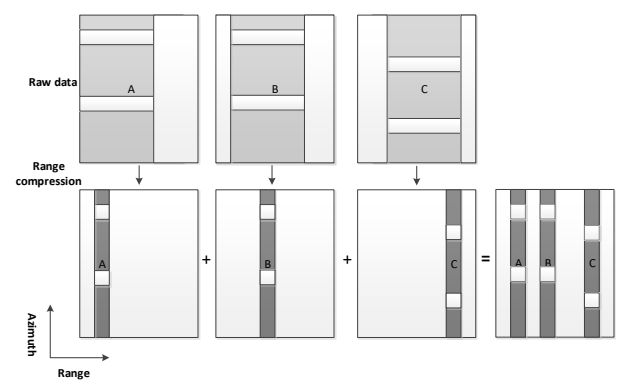

Figure 4 : Loss pattern of targets within different blind ranges. Tar-get $\mathrm{A}$ and $\mathrm{B}$ are within the same blind range, whereas target $\mathrm{C}$ is inside another one.

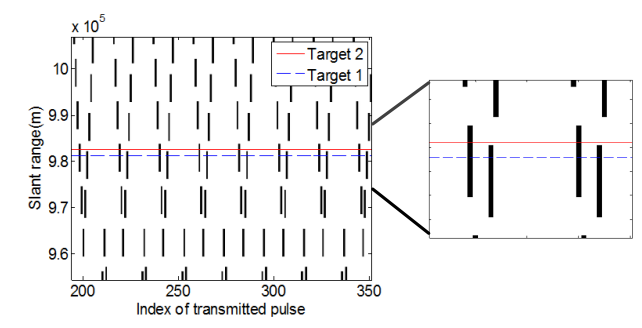

Figure 5 : Blind ranges of the PRI sequence. Red line: missing pat-tern of Target 1 . Blue dotted line: missing pattern of Target 2 .

product, $I^{\prime} \in \mathbb{C}^{N_{a} \times N_{r}}$ is the focusing image with ambiguity caused by missing samples, $N_{a}$ is the number of azimuth time or frequency pixels, $N_{r}$ is the number of range time or frequency pixels.

\subsubsection{Missing data processing method}

In Figure 3(a), it shows that before the azimuth NUFFT step, the process of recovering missing information is required in order to get a final focused image without ambiguity in azimuth. Figure 3(b) shows how an optimization problem is constructed based on the inverse process of RDA adopting NUFFT in azimuth with no need to recovering missing data. Therefore, based on Figure 3(b), the inverse imaging process of the raw data after range compression process can be formulated as

$$
S_{r c}=F_{N_{-} a}^{-1}\left(A^{*} \odot\left(F_{a} I^{\prime} F_{r}\right)\right) F_{r}^{-1}
$$

Next, we will explain how we deal with the different missing patterns of one scene on the basis of (5). For an optimization problem, we only need to know the missing positions, which is a prior information related to the specific sequence of PRI adopted. As defined in (3), $B \in \mathbb{C}^{N_{a} \times N_{r}}$ is the missing matrix, which marks the data missing position of $S_{r c}$ (i.e. echo data after range compression) by assigning value zero to corresponding position in matrix $B$, otherwise assigning value one. Therefore, the staggered SAR signal generated by targets inside blind ranges can be expressed as

$$
S_{r c}=B \odot\left(F_{N_{-} a}^{-1}\left(A^{*} \odot\left(F_{a} I F_{r}\right)\right) F_{r}^{-1}\right)
$$

If some basis in which $I$ is sparsely represented as $X=$
$\Psi(I)$ can be found, we can construct a optimization problem based on (6). We adopt $l_{1}$ as a sparse measurement, so the optimization problem is derived as

$$
\min \|X\|_{1} \text { s.t. }\left\|S_{r c}-B \odot\left(F_{N_{-} a}^{-1}\left(A^{*} \odot\left(F_{a} I F_{r}\right)\right) F_{r}^{-1}\right)\right\|_{F}^{2}
$$

where $\Psi$ is the sparsifying transform operator; $\mathcal{E}$ is the noise level, which is essential for the quality of the final focused image.

Compared to other algorithms, FISTA can be used to recover two-dimensional matrix without vectorizing the whole two-dimensional reflectivity map [8], which drastically increases the reconstruction time and requires much more memory. Therefore, in this paper we adopt FISTA to reconstruct the ambiguity-free image via (7).

\section{Performance Analysis}

\subsection{Simulation Parameter}

We perform simulation for staggered SAR with the configuration parameters given in Table $\mathbf{1}$.

\begin{tabular}{|c|c|}
\hline Parameters & Value \\
\hline Orbit height $(\mathrm{km})$ & 760 \\
\hline Sensor velocity $(\mathrm{m} / \mathrm{s})$ & 7473 \\
\hline Slant range $(\mathrm{km})$ & $837-1047$ \\
\hline Wavelength $(\mathrm{m})$ & 0.03 \\
\hline Transmit pulse width (us) & 20 \\
\hline Processed Doppler Band(Hz) & 1495 \\
\hline Mean PRF $(\mathrm{Hz})$ & 1636 \\
\hline The number of variable PRI & 21 \\
\hline Chirp bandwidth $(\mathrm{MHz})$ & 20 \\
\hline
\end{tabular}

Table 1 : Simulation parameters for staggered SAR.

\subsection{Simulation results}

The imaging results of RDA and the proposed method are shown in Figure 6. In the vicinity of the targets, obvious ambiguity can be seen if imaging directly by RDA (see left images of Figure 6(a), (b)). In contrast, the ambiguity energy reduces significantly by adopting the proposed method (see right images of Figure 6(a), (b)), which demonstrates our method is effective.

In Figure 7, by analyzing the performance of the proposed method regarding to two targets with different loss rates, it shows that when the number of iterating times reaches big enough the levels of azimuth ambiguity of the two targets with different lost rates are both well suppressed to a very low extent. In Table 2, the results of azimuth ISLR and PSLR for the four impulses responses in Figure 7 are given. It is shown that both the results of ISLR and PSLR of the two targets are greatly improved by the proposed method, compared to imaging directly, which further demonstrates the effectiveness of the proposed method. 


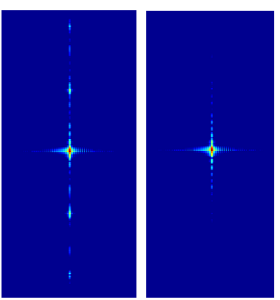

(a)

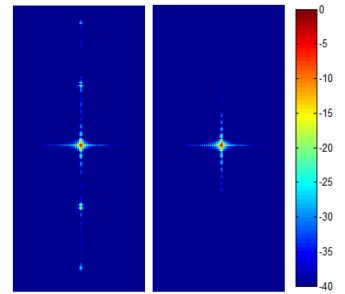

(b)
Figure 6 : Imaging results of two point-like targets. (a) Target 1. (b) Target 2. (Left of (a) and (b)) Imaging directly by RDA; (Right of (a) and (b)) Imaging by the proposed method.

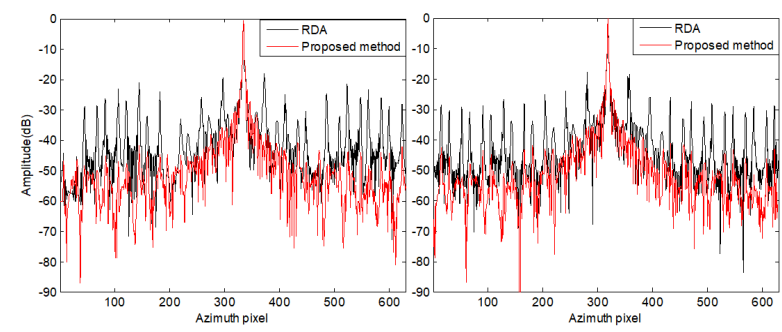

Figure 7 : Energy of the impulse response in azimuth from the point targets indicated in Figure 5. (Left) Target 1; (Right) Target 2.

To demonstrate that our method can be adopted to distributed target, we select the RadarSAT-1 image of the English Bay, which consists of part of sparse and part of dense scene. Specifically, we artificially generate the sampled data in staggered mode from this English Bay image according to parameters in Table 1. Since the scene is not sparse, we adopt the Daubechies-4 as a sparsifying operator $\Psi$, under which the scene can be transformed to be sparse. As shown in Figure 8, the imaging results of imaging directly by RDA and the proposed method are shown. It can be seen that the proposed method can effectively suppress the azimuth ambiguity caused by non-uniformly sampling and missing samples.

\section{Conclusions}

A CS imaging strategy is proposed to process the staggered SAR echo data with low oversampling factor. This strategy combines the NUFFT and the FISTA to solve an optimization problem thus to eliminate the azimuth ambiguity caused by non-uniformly sampling and the loss of echo data. The simulation imaging results show that the proposed method can effectively suppress the azimuth ambiguity in the final focused image without the recovery of the missing echo data for both point-like target and distributed target.

\section{$5 \quad$ Literature}

[1] Gebert N, Krieger G. Ultra-wide swath SAR imaging with continuous PRF variation[C]// 8th European

\begin{tabular}{|c|c|c|}
\hline Target 1 & Imaging directly & Proposed method \\
\hline ISLR & -6.7876 & -11.2995 \\
\hline PSLR & -16.5591 & -19.2731 \\
\hline Target 2 & & \\
\hline ISLR & -7.8379 & -12.0125 \\
\hline PSLR & -17.7251 & -22.3446 \\
\hline
\end{tabular}

Table 2 : Azimuth ISLR and PSLR for the four impulses responses of Figure 7.
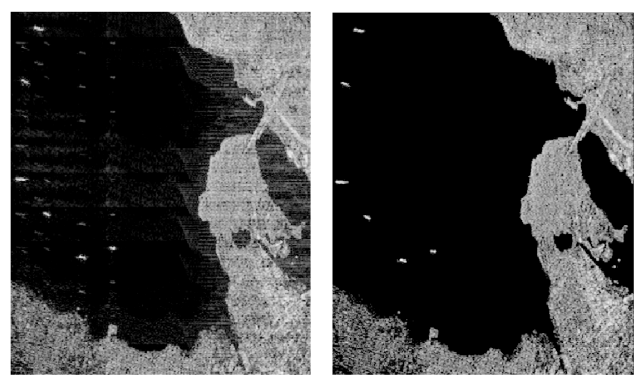

Figure 8 : Imaging results of a distributed target. (Left) Imaging directly by RDA; (Right) Imaging by the proposed method.

Conference on Synthetic Aperture Radar. VDE, 2010: 1-4.

[2] Villano M, Krieger G, Moreira A. Staggered SAR: High-resolution wide-swath imaging by continuous PRI variation[J]. IEEE Transactions on Geoscience and Remote Sensing, 2013, 52(7): 4462-4479.

[3] Luo X, Wang R, Xu W, et al. Modification of multichannel reconstruction algorithm on the SAR with linear variation of PRI[J]. IEEE Journal of Selected Topics in Applied Earth Observations and Remote Sensing, 2014, 7(7): 3050-3059.

[4] Wang X, Wang R, Deng Y, et al. SAR Signal Recovery and Reconstruction in Staggered Mode with Low Oversampling Factors[J]. IEEE Geoscience and Remote Sensing Letters, 2018, 15(5): 704-708.

[5] Pinheiro M, Prats-Iraola P, Rodriguez-Cassola M, et al. Combining spectral estimation and BLU interpolation for the reconstruction of low-oversampled staggered SAR data[C]//EUSAR 2018; 12th European Conference on Synthetic Aperture Radar. VDE, 2018: 1-6.

[6] Villano M, Pinheiro M, Krieger G, et al. Gapless Imaging with the NASA-ISRO SAR (NISAR) Mission: Challenges and Opportunities of Staggered SAR[C]//EUSAR 2018; 12th European Conference on Synthetic Aperture Radar. VDE, 2018: 1-6.

[7] Fang J, Xu Z, Zhang B, et al. Fast compressed sensing SAR imaging based on approximated observation[J]. IEEE Journal of Selected Topics in Applied Earth Observations and Remote Sensing, 2013, 7(1): 352-363.

[8] Aberman K, Eldar Y C. Sub-Nyquist SAR via Fourier domain range-Doppler processing[J]. IEEE Transactions on Geoscience and Remote Sensing, 2017, 55(11): 6228-6244. 